CAHIERS DE

NARRATOLOGIE

\section{Cahiers de Narratologie}

Analyse et théorie narratives

$33 \mid 2018$

L'Art du roman chez Umberto Eco

\title{
Narration et Conspiration. Formes de la bêtise dans le Pendule de Foucault
}

\section{Gianfranco Marrone}

\section{(2) OpenEdition}

\section{Journals}

Electronic version

URL: http://journals.openedition.org/narratologie/7994

DOI: 10.4000/narratologie.7994

ISSN: 1765-307X

Publisher

LIRCES

\section{Electronic reference}

Gianfranco Marrone, "Narration et Conspiration. Formes de la bêtise dans le Pendule de Foucault », Cahiers de Narratologie [Online], 33 | 2018, Online since 23 July 2018, connection on 15 November 2019. URL : http://journals.openedition.org/narratologie/7994 ; DOI : 10.4000/narratologie.7994

This text was automatically generated on 15 November 2019.

Article L.111-1 du Code de la propriété intellectuelle. 


\title{
Narration et Conspiration. Formes de la bêtise dans le Pendule de Foucault
}

\author{
Gianfranco Marrone
}

« Era giunto il momento di dare una funzione al

Pendolo» (Eco)

\section{Entre théorie et roman}

1 «Ce qu'on ne peut théoriser, il faut le narrer ». Voici la périphrase d'un des aphorismes les plus célèbres de Ludwig Wittgenstein, qui se trouve sur la page de garde de la première édition du Nom de la rose d'Umberto Eco (1980) : clé pour comprendre l'itinéraire et le sens profond du travail d'un sémioticien qui venait d'écrire - et de publier - de la littérature en tant que telle ${ }^{1}$. D'habitude, c'est le texte qui détermine son paratexte (Genette 1987), mais c'est possible, parfois, que ce soit aussi le contraire : comme dans ce cas-là, où la couverture du premier roman d'un essayiste devient prophétique de l'œuvre future de l'auteur dans sa totalité, de ses tendances ambivalentes, des oscillations entre la parole sémiotique et la parole littéraire.

Donc, certaines des asymétries entre le travail théorique et celui de l'écriture littéraire, chez Eco, doivent être interprétés comme les traces d'une progressive intégration vers un horizon intellectuel commun: pas dans le sens trivial de la "traduction » d'un noyau conceptuel dans un roman de fiction, mais, plus précisément, dans celui d'un ajustement mutuel et d'un achèvement partiel entre les deux univers de la théorie sémiotique et de la pratique littéraire. Fabbri (1989), à ce propos, a remarqué comment certaines des questions ouvertes de la théorie d'Eco, par exemple celle du texte esthétique, ont trouvés dans ses romans des «solutions figuratives " plutôt que des correspondances absolues ${ }^{2}$. Ainsi, la théorie du comique énoncée plusieurs fois dans les écrits théoriques ne coïncide pas entièrement avec l'image du rire qui émerge à partir des discussions entre les personnages dans le Nom de la rose. De même, les enquêtes sur 
la logique de l'abduction ne peuvent pas être appliquée sic et simpliciter à la pratique de la détection du protagoniste du roman Guillaume de Baskerville. Ou, encore, la classification des modes de production des signes proposée dans le Trattato di semiotica generale ne constitue pas une réponse automatique au grand nombre des traces, indices, signaux, symptômes distribués au cours du roman. Entre les deux aspects, il y a un reste, une fracture, une différence : c'est ce qui a permis à Eco de jouer le double travail de réflexion et d'écriture sur la base d'un seul problème conceptuel ${ }^{3}$.

3 Pour le Pendule de Foucault c'est presque la même chose : ce n'est pas possible de penser que ce roman très complexe et articulé puisse être réduit à la représentation littéraire du problème de l'irrationalisme sur lequel Eco a beaucoup travaillé, dans ses textes sémiotiques, au cours des années de l'écriture du roman ${ }^{4}$. L'histoire de la Conspiration qui se développe au cours de la narration romanesque à partir de l'aventure des Templiers n'est pas la représentation idéalisée de l'hermétisme et de l'occultisme médiéval et moderne. Il est plutôt la reconstruction figurative d'une erreur intellectuelle qui engendre un délire d'interprétation. Le Plan existe parce que les trois protagonistes, enchantés par la « forêt des ressemblances », décident d'en reconstruire la conjecture progressive jusqu'à s'identifier avec elle. Le Secret qui serpente le long des événements historiques a sa raison d'être dans la croyance au Secret lui-même. C'est la « crédulité » comme " passion de l'esprit »-posée par dénégation dans l'ouverture du livre (47) $)^{5}$ à être la porte d'entrée à la folie individuelle et collective, qui fait son chemin dans la trame du roman ${ }^{6}$. Et le fait que, à la fin, l'enquête de Casaubon, Belbo et Diotallevi se révèle efficace (le soir du 23 Juin 1984 aura lieu effectivement la réunion secrète au Conservatoire des Arts et Métiers à Paris) ne fait que confirmer la capacité de cette croyance d'influencer les choix existentiels et intellectuels, et de susciter de nouvelles croyances... au point d'« engendrer » la réalité. Dans le délire commun, on va perdre la différence entre réel et imaginaire, raison et déraison. Au point que cet irrationalisme, qui d'abord pourrait être considéré comme une tendance à détecter et combattre, devient beaucoup plus clair et déterminé; ses charmes envahissent les critères stricts d'une logique cognitive et trouvent consolation dans les états affectifs des sujets.

D'où l'importance, dans le roman, d'une théorie, abstraite et figurative, de la stupidité. Cette notion, en effet, n'est pas une notion au sens strict. Et précisément en raison de sa complexité inhérente, curieux mélange des forces et des facultés diverses et parfois contradictoires, la bêtise réarticule la réflexion sur les mécanismes de la cognition et sur les critères de l'argumentation, en montrant aussi les limites et les risques d'une théorie de la connaissance et d'une logique trop restreintes.

\section{Une typologie idéale}

5 Dans le dixième chapitre du roman (70-76) il y a une typologie des stupides à laquelle il vaut la peine de réfléchir avec une certaine attention. Il s'agit de la première rencontre entre Casaubon, qui prépare une thèse sur les Templiers, et Belbo, éditeur expert. Belbo illustre à Casaubon sa méthode pour détecter immédiatement les fous qui écrivent des livres sur les Templiers et essayent de les publier ${ }^{7}$.

6 Donc. Au monde il y a les crétins, les imbéciles, les stupides et les fous. Chaque personne participe de différentes manières et temps à ces quatre catégories idéales : «la personne normale est celle qui mêle en une mesure raisonnable toutes ces 
composantes, ces types idéaux» (71), et le génie, c'est celui qui fait jouer une composante de façon vertigineuse, en la nourrissant avec les autres composantes.

Il y a d'abord le crétin. A mi-chemin entre l'aliéné mental et le clown, il a peu à voir avec le véritable stupide, et moins encore avec le postulant en embuscade dans les maisons d'édition : «Le crétin ne parle même pas, il bave, il est spastique. Il plante son sorbet sur son front, par manque de coordination. Il prend la porte-tambour en sens contraire » (72). Sa particularité, sa niaiserie s'installe précisément dans sa capacité à faire des choses que les autres ne savent pas faire, comme entrer « la porte-tambour en sens contraire " (58). Il s'agit donc d'une bêtise qui met en jeu surtout la dimension corporelle, somatique du sens.

8 En ce qui concerne l'imbécile, lui aussi convoque une composante actionnelle, mais il s'agit d'un type bien plus complexe. L'imbécile « est un comportement sociale ». Il est le gaffeur, celui qui parle hors de son tour, l'inopportun qui intervient justement pour dire exactement ce qu'on n'attend pas de lui.

«L'imbécile est fort demandé, surtout dans les occasions mondaines. Il met tout le monde dans l'embarras, mais ensuite il offre matière à commentaires. Dans sa forme positive, il devient diplomate. Il parle hors de son verre quand ce sont les autres qui ont fait une gaffe » (72).

9 Animal des salons, il a des ascendances littéraires très fortes : les maisons Verdurin et Guermantes sont ses domiciles préférés. Pour cela, il s'agit d'une espèce purement bourgeoise, « une race en voie d'extinction » (72). C'est ce qui est à l'œuvre ici, une dimension pragmatique, sociale, narrative de la signification.

Les erreurs de raisonnement caractérisent plutôt le véritable stupide : «C'est lui qui dit que tous les chiens sont des animaux domestiques et que tous les chiens aboient, mais que les chats aussi sont des animaux domestiques et donc qu'ils aboient» (73)... Ses fautes sont des erreurs de logique formelle, qui peuvent aussi formuler, mais par hasard, une certaine vérité («tous les Athéniens sont mortels, tous les habitants du Pirée sont mortels, et donc tous les habitants de Pirée sont athéniens » (73). «C'est un maître ès paralogismes" (73). D'où la difficulté à le reconnaître: «L'imbecille lo riconosci subito (per non parlare del cretino), mentre lo stupido ragiona quasi come te, salvo uno scarto infinitesimale » (59). Et de là, les dangers qu'il provoque presque inévitablement. De là, les dangers qu'il provoque presque inévitablement : «On publie beaucoup de livres de stupides parce que, de prime abord, ils nous convaignent. Le lecteur d'une maison d'édition n'est pas tenu de reconnaître le stupide. L'académie des sciences ne le fait pas, pourquoi l'édition devrait-elle le faire?» (73). Voilà donc comment se manifeste la bêtise dans une dimension spécifiquement cognitive du sens.

11 Enfin, le fou. Contrairement au stupide, qui par le biais d'une « logique biscornue » (75) cherche toujours à prouver son point de vue, le fou est « un stupide qui ne connaît pas les trucs ", qui « ne se soucie pas de logique » et, par conséquent, " procède par courtcircuit » (75).

« Le fou a une idée fixe, et tout ce qu'il trouve lui va pour la confirmer. Le fou, on le reconnaît, à la liberté qu'il prend par rapport au devoir de preuve, à sa disponibilité à trouver des illuminations. Et ça vous paraîtra bizarre, mais le fou, tôt ou tard, met les templiers sur le tapis » (75).

12 Même le fou donc (installé plutôt dans la dimension passionnelle de la signification), compte tenu de ces caractéristiques évidentes, est comme le crétin et l'imbécile, immédiatement reconnaissable. Or, cette petite taxonomie montre clairement que le 
seul élément de la classification qui n'a pas des aspects positifs est le stupide. Si les deux types extrêmes - la personne normale et le génie - semblent ne pas avoir des côtés négatifs, et si le crétin, l'imbécile et le fou, dans certaines circonstances, peuvent montrer des comportements positifs, le stupide est la personne la plus perfide, parce qu'il se masque souvent en tant que type intelligent: et justement grâce à cette capacité de camouflage, il est encore plus dangereux que les autres.

élements virtuels qui composent cette typologie sont destinés à revenir au cours de l'histoire ultérieure du roman, où ils trouvent une mise en œuvre très précise. Il s'agit bien sûr des "idéaltypes " - comme Belbo le rappelle -, et très souvent il arrive que, chez quelques personnes et dans certaines situations, on peut trouver mélangés et réarrangés pour l'occasion plus d'un type à la fois. Il semble indéniable que, si, à ce moment du roman, l'intérêt pour les Templiers est une prérogative du fou, dans le suite de l'histoire le rôle du héros sera interprété par le stupide : par quelqu'un de bête qui, dans sa métamorphose continue, prendra le masque du fou, puis de sage, puis encore d'imbécile et ainsi de suite; et, à la fin, il imprégnera toute capacité de raisonner avec ses paralogismes, ses conjectures, ses réfutations. Si le Nom de la rose est l'histoire d'un raisonnement efficace qui, après quelques doutes, conduit l'enquête policière jusqu'à la véritable solution, le Pendule de Foucault suit le chemin inverse : il montre les moyens et les mécanismes par lesquels la lucidité rationnelle peut conduire, presque sans le savoir, à la frénésie débridée. Il est l'histoire de la stupidité victorieuse, que seule une régénération entre le tragique et le grotesque puisse partiellement surmonter.

\section{Le filtre de Pilade}

14 Il ne faut pas oublier, pourtant, que cette typologie est énoncée dans le cours d'une conversation chez Pilade, un vieux bar des canaux milanais, c'est-à-dire un lieu où se sont succédées, au fil des ans, des idéologies très diverses, une sorte de miroir des attitudes et des coutumes de plusieurs générations d'intellectuels et d'écrivains. "Je pourrais écrire l'histoire politique de ces années-là en enregistrant les temps et les manières dont l'on passa graduellement de l'étiquette rouge au Ballantine de douze ans d'âge et enfin au malt. » (62). Et la haute quantité d'alcool bue, ce jour-là, par les deux interlocuteurs, ne peut que créer un certain embarras au lecteur à la recherche des vérités absolues. Bientôt, la subtilité des distinctions proposées par Belbo et partagées par Casaubon cède la place à des possibilités combinatoires très larges. La théorie de paralogismes envahit peu à peu toute l'histoire de la philosophie: "une lutte entre stupides" qui est très amusante pour ce Dieu dont la démonstration d'existence provoque une dérive d'arguments stupides. Jusqu'à ce que, de façon prévisible, le tribunal de la logique commence à se juger lui-même: "nous sommes entourés de stupides. ». D'où l'émergence d'une sorte de relativisme de nature anthropologique :

«"-Et si le stupide était vous ?" "Je serais en bonne et séculaire compagnie." “-Eh oui, la stupidité nous entoure. Et peut-être par un système différent du nôtre, notre stupidité est leur sagesse. Toute l'histoire de la logique consiste à définir une notion acceptable de stupidité. Trop immense. Tout regard penseur est le stupide d'un autre. " (75). " «"La pensée comme forme cohérente de la stupidité"; "Non la stupidité d'une pensée est l'incohérence d'une autre pensée." “-Profond. Il est deux heures du matin, d'ici peu de temps Pilade va fermer et nous ne sommes pas arrivés aux fous. " (75)» 
La raison du système produit ses propres sommeils. Les principes logiques de base, ceux sur lesquels a été fondé le rationalisme occidental depuis Aristote, sont dépassés par des obstacles importants et produisent des paradoxes inhérents aux mêmes arguments dont ils sont les garants. S'insinue le soupçon qu'ils ne peuvent pas être les garants de la vérité du discours dit rationnel, parce qu'ils ne sont pas la seule et unique source de certitude. Le suite du roman va montrer, parallèlement, combien les soidisant évidences irrationnelles, mythiques, fantastiques - comme les plans des « diaboliques » et les conséquentes théories de la Conspiration - possèdent une logique, sinon rigoureuse comme celle syllogistique, certainement plus efficace: celle de l'analogie. Bien sûr, on peut dire que, au-delà de l'excitation alcoolique du moment, les partitions de la bêtise proposées par Belbo sont basées sur une pratique éditoriale (et, en général, sur une connaissance du monde) qui a été développée au fil du temps par l'expérience. Mais il est également vrai que la complaisance apodictique avec laquelle la typologie a été énoncée laisse plus d'un doute au lecteur, et elle le pousse à vérifier dans quelle mesure cette scène peut être considérée comme une preuve fidèle de la théorie des types de la stupidité. En d'autres termes, c'est le roman lui-même qui, si d'un côté, il invite son lecteur (victime) à assumer des types idéaux de stupidité proposés par Belbo, d'un autre côté, il suggère au lecteur (critique) de les considérer avec une certaine perplexité 8 . Le Pendule de Foucault est le chemin d'apprentissage qu'il faut parcourir pour comprendre comment toute sorte de barricade érigée entre raison et déraison, délire et argumentation, raisonnement et sophisme est soumise à des changements constants de l'esprit, en étant, en dernier lieu, une source continuelle d'incertitudes et de doutes nécessaires à la préservation des véritables connaissances et de la liberté de pensée. Les oscillations du pendule de Léon Foucault au Conservatoire d'art et métiers peuvent, de ce point de vue, bien représenter les rythmes d'un doute méthodique, qui a tendance à se méfier de toute vérité définitive, de toute certitude, de tous principes établis - au nom desquels, souvent, viennent s'exercer l'autorité et la violence. Et si l'alcool est un indicateur de la faiblesse de la typologie de Belbo, le cours du roman, avec ses personnages et leurs argumentations très souvent pataphysiques, en est une preuve supplémentaire.

\section{Le Plan et le Livre}

«In quei giorni felici credevo che la stupidità creasse enigma. L'altra sera pensavo che gli enigmi più terribili, per non rivelarsi tali, si travestono da follia. Ora penso invece che il mondo sia un enigma benigno, che la nostra follia rende terribile perché pretende di interpretarlo secondo la propria verità » (82, c'est moi qui souligne).

Arrivé à la fin de son voyage d'apprentissage ${ }^{9}$, Casaubon réfléchit sur l'itinéraire qu'il a parcouru et sur les croyances qui se sont progressivement mises en place. Parmi elles, l'idée de la bêtise est souvent reformulée à partir du pli des événements, qu'elle tend au même moment à orienter. Suivre les étapes de l'histoire racontée par le roman signifiera donc suivre le faire et le refaire de l'image de la stupidité.

En particulier, la structure du récit dit de l'enthousiasme des protagonistes pour le Plan des Templiers. Dans l'articulation et dans le chevauchement de l'ironie et de crédulité dont le rythme est marqué par la succession des chapitres - il est possible d'inscrire l'échec de la rationalité du modus ponens d'Aristote, en faveur du principe universel de l'analogie, ou, en d'autres termes, la victoire d'une stupidité exubérante sur les pauvres 
attractions d'une intelligence en tant que telle. Si Belbo, Diotallevi et Casaubon, chacun à partir de leurs propres expériences et croyances, s'approchent du monde et de l'histoire de l'occultisme sur la base de leurs certitudes rationalistes, la suite de l'histoire va les obliger à reconsidérer leurs positions et, pire, à tomber dans des formes de bêtise ou de folie stigmatisées par Belbo, ce soir-là, chez Pilade. De la reconstruction pataphysique d'un Secret qui a été passé à travers de l'histoire, on passe progressivement, avec des moments différents pour les trois personnages, à l'engouement pour la Conspiration et à la croyance en elle. On comprend ainsi comment la typologie de Belbo est doublement fallacieuse. Tout d'abord, parce qu'il est impossible de distinguer, sinon à un niveau idéal et abstrait, le comportement (du crétin et de l'imbécile) du raisonnement (du stupide et du fou). En second lieu, parce que les armes du syllogisme ne peuvent pas, en tant que telles, faire face à la puissance de la stupidité, ni même en motiver les succès.

Pourquoi tout cela? Pourquoi le plan fascine les protagonistes au point d'envahir entièrement leur conscience - et, dans le cas de Diotallevi, le même corps ? Dans quels termes a lieu ce passage d'un snobisme tout à fait intellectuel à la crédulité la plus naïve ? La réponse est précisément à chercher dans la structure du roman. Il n'est pas difficile de s'apercevoir que, en fait, l'écriture du livre coïncide avec la construction du Plan. Toute la fabula n'est pas autre que l'explication d'une sorte d'ingénierie narrative. Les actions des protagonistes sont, on pourra le dire, performatives: les mots se confondent avec les choses, le langage est la seule forme possible de comportement. Les personnages vivent pour le Plan et vivent par le Plan; et le racontant, ils le créent; en croyant en lui, ils finissent par l'actualiser. Pas de séparation entre langue et métalangage, réalité et fiction, vérité et croyance. La forme du roman, la forme-roman, assure leur mélange infini. Et les personnages ne peuvent qu'en subir les conséquences : ils imitent dans la fabula ce qui se passe dans le récit.

19 Si on observe attentivement les attaques et les fermetures des chapitres, par exemple, on remarquera que chaque fois que l'énonciateur va insérer un nouveau morceau du puzzle (des Templiers à la Kabbale, aux rose-croix, aux jésuites, aux meurtriers, aux nazis et ainsi de suite) cela implique une rapide digression, soit du narrateur soit des personnages, où on discute les modes de construction du Plan, à savoir les procédures narratives qui sont mises en place, afin qu'il puisse être considéré par les personnages $^{10}$. De même, les fichiers de Abulafia (l'ordinateur), les flashbacks sur l'enfance de Belbo, les relations entre Casaubon et Amparo, entre Casaubon et Lia, entre Belbo et Lorenza, les interventions de Aglié et de Garamond, sans oublier les scènes rituelles au Brésil et dans le Piémont, bref, tout le contexte dans lequel les trois protagonistes s'agitent ne sert qu'à balayer les rythmes réguliers des différentes phases de la construction du Plan et les degrés d'adhésion à lui. De plus, la rhétorique avec laquelle sont racontés les différents moments du Plan n'est pas accidentelle : elle vise à passionner l'interlocuteur, à le persuader de la grandeur incontestable des événements décrits, à savoir du Plan même. Donc, si Casaubon raconte l'aventure des Templiers " con toni tra il western e il cartone animato » $(71,77)$, à sa façon le colonel Ardenti présente sa thèse avec un " senso del teatro " très efficace $(102,118)$. Pour ces raisons, le lecteur (naïf) a l'impression de glisser progressivement dans l'atmosphère hallucinatoire de l'histoire, accompagnée d'un sentiment calculé de malaise et d'ennui que la longueur exténuante du récit ne peut pas ne pas provoquer. La construction du Plan - l'écriture du livre - procure une gêne quelque peu analogue à ce qu'on ressent en lisant des partouzes sadiennes: la répétition obsessionnelle des mêmes gestes et des 
mêmes situations produit ce que l'on pourrait appeler un 'effet de surréel', ou, si l'on veut, une sorte de distanciation organisée et constante. L'adhésion surveillée du lecteur à la fiction narrative est en même temps la croyance des personnages envers la Conspiration : "Credo - dit rétrospectivement le narrateur Casaubon - che non ci sia più differenza, a un certo punto, tra abituarsi a fingere di credere e abituarsi a credere » (367). Le problème est donc l'impossibilité constitutive de localiser le « certo punto » où a lieu la transition entre la fiction et la réalité. Chose qui vient de la nature même de la logique sur laquelle se fonde l'idée de la Conspiration et le comportement des soi-disant « diaboliques ».

\section{La logique de la Conspiration}

20 La logique du récit coïncide avec celle de la Conspiration. Il s'agit d'une logique stupide mais efficace, capable de réchauffer les cœurs et de conduire à des actes de foi incongrus et à la violence conséquente. Immédiatement après la narration de l'histoire des Templiers, Casaubon en tire la bonne morale, renvoyant à la typologie de Belbo :

«-Je crois que c'est un peu votre histoire de l'autre soir. Toutes leurs vicissitudes ne sont qu'un syllogisme contourné. Comporte-toi en stupide et tu deviendras impénétrable pour l'éternité. (...) chaque fois qu'un poète, un prédicateur, un chef, un mage ont émis d'insignifiants borborygmes, l'humanité met des siècles à déchiffrer leur message. Les Templiers restent indéchiffrables à cause de leur confusion mentale. C'est pour cela ça que tant de gens les vénèrent. » (113)

21 Le résultat pragmatique du paralogisme du stupide ou le " court-circuit $»^{11} \mathrm{du}$ fou est la folie de ceux qui, comme Belbo, décident de faire un changement dans leur existence malheureuse, ou de quelqu'un comme Aglié qui n'est plus capable de se donner une identité en se croyant immortel. Mais il est aussi la folie collective qui, tout au long de l'histoire, donne lieu à des meurtres et des massacres, aux inquisitions, aux tortures, aux autodafés, même à l'holocauste. Le résultat, en bref, est de mélanger réalité et fiction, vérité et mensonge. Mais comment fonctionne, du point de vue formel, la logique de la stupidité ? C'est à Casaubon, à la fin de l'histoire, qu'incombe de reconstruire les trois règles de base qui sous-tendent le mode de penser non rationaliste des diaboliques. Règles que lui, Belbo et Diotallevi ont embrassé, et ont appliqué avec une telle perfection, au point de construire un Plan qui a convaincu et séduit aussi les diaboliques ${ }^{12}$.

La première règle est celle de l'analogie universelle, fondée sur le principe que "qualsiasi cosa è simile a qualsiasi altra sotto un certo rapporto" $(489)^{13}$. Les combinaisons d'événements, des choses et des gens ne sont pas faites selon les lois de causalité, ne présupposent pas des hiérarchies logiques ou linguistiques, mais sont basées sur des ressemblances vagues de toute sorte : «le connessioni ci sono sempre, basta volerle trovare" (180). Ainsi, l'utilisation aveugle de l'analogie conduit à l'abolition de la séparation rhétorique entre littéral et figuré :

«Tutti stavamo lentamente smarrendo quel lume intellettuale che ci fa sempre distinguere il simile dall'identico, la metafora dalle cose, quella qualità misteriosa e folgorante e bellissima per cui siamo sempre in grado di dire che un tale si è imbestialito ma non pensiamo affatto che gli siano cresciuti peli e zanne, e invece il malato pensa 'imbestialito' e subito vede colui che abbaia o grufola o striscia o vola » (367). 

logique aristotélicienne sur laquelle est basé le rationalisme occidental. Face aux "catene puntigliose ed ottuse» (313) de ce dernier, la logique de la stupidité est présentée comme une non-logique ; et dans cette apparence réside justement sa force, sa capacité à se replier en bon ordre, à ne pas montrer ses cartes et à agir subrepticement dans les subtilités du pseudo raisonnement syllogistique. Le maitre de cette ancienne sagesse est certainement Aglié, qui ne lésine pas sur ses enseignements : "Il vero esoterismo - dit-il - non ha paura dei contrari» parce que "qualsiasi errore può essere il portatore misconosciuto della verità » (268). De même, «il saggio non è colui che discrimina, è colui che mette insieme i brandelli di luce da dovunque provengano" (144). A Agliè s'oppose Lia, personnage qui, tout en restant stratégiquement dans l'ombre, développe une expérience positive de lucidité intellectuelle. Au délire d'interprétation de Casaubon, qui révèle à son partenaire ses découvertes au sujet du Plan, Lia fait contraste, avec un scepticisme typique de la personne sensée, et elle construit une nouvelle interprétation du message de Provins, comme s'il s'agissait d'une liste de blanchisseuse : «per dimostrarmi - dit Casaubon che sono stupido » (419). A la chute progressive des trois personnages dans le monde de l'occulte, Lia répond avec une proposition de vie tout à fait normale : elle attend un bébé. La Chose qui se déplace dans le ventre de Lia est pour Casaubon un rappel constant à la lucidité, au côté non obscur de l'existence. elles génèrent aussi la gamme de leurs contenus possibles. A la nonchalance de la méthode analogique, il faut nécessairement superposer un paramètre profond, qui permet les connexions de tout avec tout. Il faut soupçonner, donc, selon cet état d'esprit, l'existence d'un Plan caché, qui justifie les similitudes à première vue 
incongrues entre les choses, qui explique la dialectique sapientiale entre la réalité et l'apparence (166). "Quando ci si mette in uno stato di sospetto non si trascurerà più nessuna traccia » (303); on ira ainsi à la recherche d'une Conspiration qui puisse préserver un secret, un secret "pur", sans objet, qui ait comme but principal de susciter une croyance générale dans le Plan. «La connessione - explique Belbo cambia la prospettiva. Induce a pensare che ogni parvenza del mondo, ogni voce, ogni parola scritta o detta non abbia il senso che appare, ma ci parli di un Segreto. Il criterio è semplice : sospettare, sospettare sempre » (300). Mais le secret n'a pas une syntaxe ou une sémantique: il a seulement une pragmatique. C'est la croyance dans le secret qui produit le secret $m e ̂ m e^{15}$. «Il vero iniziato è colui che sa che il più potente dei segreti è un segreto senza contenuto, perché nessun nemico riuscirà a farglielo confessare, nessun fedele riuscirà a sottrarglielo » (492). Si on se comporte comme s'il existait un Plan, le Plan assume réalité et vérité: une logique abstraite a donc des résultats significativement tangibles. Dans ce cas - signal de la stupidité suprême - ce qui vient après rend possible ce qui vient avant.

\section{Abulafia et Lorenza}

«Macchina stupida, non ti emozioni neppure al pensiero di Lorenza » $(40)^{16}$. Déconcerté par le fait qu'il ne possède pas le mot de passe pour entrer dans le "ventre" de l'ordinateur Abulafia, Casaubon a un moment d'égarement éclairant ${ }^{17}$. Chez Belbo, sa dépendance $d u$ " truc-confesseur » est difficile à concilier avec l'émotion érotique, ainsi que son désir du passé, qui ne se reflète pas dans sa soif de rigueur logique. En d'autres mots, la passion et la raison ont dans son âme des liaisons dangereuses, elles contrastent entre elles de façon transparente : ce qui provoque de nombreux troubles psychologiques au pauvre éditeur déçu par sa vie dépourvue d'héroïsme.

Belbo est sans aucun doute le personnage central du livre, et non pas tant - comme certains ont voulu le comprendre - pour son analogie possible avec l'auteur, mais plutôt parce qu'en lui est concentrée la majorité des forces dont les vecteurs se croisent tout le long du roman. Ses contradictions psychologiques et existentielles - typiques d'une génération et peut-être d'une société - découlent parallèlement au récit : le jeu de rôle (d'une part les diaboliques, avec leur patrimoine historique et culturel, de l'autre la raison, avec sa rigueur brillante) disparait progressivement dans un mélange généralisé de similitudes et d'analogies. La réforme du savoir conçue avec Diotallevi est essentiellement le Plan avant son actualisation : la Faculté de Pertinence Comparée que les deux imaginent (66-67) ne fait qu'anticiper les deux collections éditoriales (Isis Dévoilée et Hermetyca), qui le conduiront sur le chemin des diaboliques. De même, son envie de trouver des stupides partout (73) obtient l'effet inverse : celui de perdre la tête jusqu'à l'infatuation et le sacrifice final.

30 Sa classification des types de bêtise, donc, semble être générée par l'esprit de quelqu'un qui a tout compris sur la vie, et organise sa propre vie en conséquence. Mais, dans cette classification, se cache un problème sans solution. Belbo voit le monde sub specie éditoriale, et pense que le microcosme de la maison d'édition (où il y a une lutte quotidienne entre ceux qui veulent publier des livres absurdes et qui, de l'autre côté, tentent d'entraver - avec des compromis inévitables - la propagation de la stupidité) est applicable au macrocosme externe (où, pourtant, on se comporte sur la base d'autres codes, très divers). Par conséquent, en dépit de ses mêmes remarques, c'est lui-même 
qui encourage l'application constante et obsessionnelle de sa typologie des stupides à des hommes et des choses qui ne le sont pas nécessairement. De même, les difficultés dans sa relation avec Lorenza Pellegrini ne dépendent pas de contrastes triviaux entre eux. Si on observe les étapes infinitésimales du changement d'humeur de Belbo déceptions, honte, mensonges, plaisanteries et désirs de toutes sortes - on rencontre en profondeur une sorte de motif constant: c'est l'impossibilité de concevoir intellectuellement quelque chose qu'en quelque sorte, pourtant, il perçoit : la raison et la passion, le dynamisme et l'émotion. La machine et la pulsion ne sont pas antithétiques; elles ne constituent pas des mondes séparés ou, avec un terme médiéval, incompossibles. Elles sont étroitement liées, communiquent entre elles sans cesse, en échangeant les rôles l'une avec l'autre. Abulafia ne s'excite pas face à Lorenza, mais il s'ouvre à ceux qui lui communiquent leur détresse : le mot-clé pour y accéder c'est «non » (41). Et si Lorenza trouble Belbo, c'est surtout par la façon dont elle joue au flipper, en agitant son entrejambe (177). Entre la machine et le corps se produit une symbiose qui, si elle n'est pas pensable avec les modèles de la logique formelle, est pourtant à l'origine de nombreuses actions humaines et sociales.

31 En conséquence, les crises périodiques de Belbo et les débordements qui en découlent dans les fichiers de Abulafia ne sont pas simplement le résultat de son manque de leadership. Dire, comme le fait Casaubon, que «il suo depresso libertinismo intellettuale celava una disperata sete di assoluto » (51) c'est simplifier son caractère, réduire à de naïves contradictions non résolues une psyché qui doit être lue comme une querelle profonde entre des logiques opposées: celle du syllogisme, d'un côté, et celle du paralogisme, de l'autre, celle du savoir-faire et celle du fou. Le principal défaut de Belbo, probablement, est de radicaliser constamment les positions, d'agir plus par principe qu'avec du bon sens, en prenant au sérieux le contraste irrémédiable entre les deux logiques, en adhérant d'abord à l'une et puis à l'autre. Et il trouvera la mort justement «per provare a se stesso e agli altri che, anche in difetto del genio, l'immaginazione è creatrice» (475). La parabole de Belbo est exemplaire parce qu'il n'est pas capable de discerner entre la surface et la profondeur, entre l'apparence et la réalité. En faisant ainsi le jeu des diaboliques.

La seule façon d'en sortir serait de rester en équilibre sur le bord de la fascination, de demeurer dans cette limite au-delà de laquelle l'intérêt devient adhésion. Selon Casaubon, c'est la façon que Belbo en quelque sorte utilise : «Mi ero chiesto sovente, lavorando con lui, perché accettasse quella situazione. [...] Avevo creduto a lungo che lo facesse perché poteva coltivare i suoi studi sulla stoltezza umana. Quella che lui chiamava stupidità, il paralogismo imprendibile, l'insidioso delirio travestito da argomentazione impeccabile, lo affascinava - e non faceva che ripeterlo" (202). Si on ne peut pas jouer la trompette, on peut en tout cas jouer le génie, « lo strumento più stupido di tutta la banda » (261). Si on ne peut pas écrire sa propre histoire, il faut réécrire celle des autres. En d'autres termes, si on ne peut pas lutter contre la stupidité (parce que, dans tous les cas, elle gagne), on pourra jouir du charme qui, selon Flaubert, s'en émane toujours. Charme subtil et envoûtant qui peut conduire, s'il est poursuivi à chaque moment, à la perte de soi : la limite est dépassée, l'équilibre perdu ; lutter pour jouer en tous cas de la trompette, abandonner Abulafia pour Lorenza est trop risqué. On perd son esprit et son sens du jeu. On devient adepte de ses propres fictions. On cherche des significations où il n'y en a pas, on tombe inévitablement dans le sans fond de la stupidité - dans tous ses états. 


\section{BIBLIOGRAPHY}

Bernoussi, Mohamed. 2017. Umberto Eco sémioticien et romancier, Sesto San Giovanni, Mimesis.

Eco, Umberto. 1980. Il nome della rosa, Milano, Bompiani.

-1988 . Il pendolo di Foucault, Milano, Bompiani.

-1989. « Il testo ne sa di più », Nuove Effemeridi, II, 6.

-1990 . I limiti dell'interpretazione, Milano, Bompiani.

-1998. Six promenades dans les bois du roman et d'ailleurs, Paris, Grasset.

Fabbri, Paolo.1989. « Le dedale dans le texte », Magazine littéraire février.

Fabbri, Paolo \& Petitot, Jean (éds.). 2000. Au Nom di Sens, Paris, Grasset.

Genette, Gérard. 1987. Seuils, Paris, Seuil.

Lorusso, Anna Maria. 2008. Umberto Eco, Rome, Carocci.

Magli, Patrizia, Manetti, Giovanni \& Violi, Patrizia (éds.). 1992. Semiotica: storia teoria

interpretazione, Milan, Bompiani.

\section{NOTES}

1. Pour une bonne synthèse du travail théorique d'Umberto Eco cf.,Bernoussi (2017); Lorusso (2008). Cf. aussi Magli, Manetti \& Violi éds. (1992) et Fabbri \& Petitot éds. (2001)

2. «La réponse à ces questions Eco l'a, sans doute, déjà donnée dans ses textes de fiction (il suffit de penser à l'assassin aveugle et à l'enquêteur myope se poursuivant dans la nuit labyrinthique d'une bibliothèque dans Le nom de la rose !). Cette écriture virtuose peut être lue comme un Gedankenexperiment, qui emploie de façon théoriquement inédite la langue des "genres (dits) mineurs" et du pastiche. Encore une fois contre Croce, qui opposait dans les textes mêmes de la Grande Littérature moments poétiques et simple structure, Eco a un goût prononcé pour les textes paralittéraires. Il se sert de leur rationalité propre pour esquisser une "solution figurative" aux problèmes du sens. Le Policier se fait parabole cognitive pour les stratégies inférentielles de l'abduction (dans Le nom de la rose) ; le Roman de Mystère, du genre Mystère de Paris, devient l'allégorie des jeux de simulacre et du secret (dans Il Pendolo di Foucault)» (Fabbri 1989).

3. . Après tout, l'auteur a toujours refusé d' "expliquer ses romans ", et a pris souvent des positions contre toute assimilation entre le fiction et non-fiction. Selon Eco (1989), la seule distinction possible entre les textes artistiques et les textes scientifiques est "pragmatique ", à savoir elle réside «dans un autre type d'engagement à défendre le droit de l'interprétation du texte ». Donc, si le texte créatif «pose un nœud problématique » et le passe au lecteur qui doit l'interpréter dans la mesure où c'est le même texte à poser ses limites herméneutiques, le texte scientifique vise à "éliminer le nœud» et défend une interprétation unique de son discours. Encore une fois: "L'engagement du texte créatif [...] est de garder intacte l'oscillation, une densité d'ambiguïté ; pour lui il s'agit est d'essayer de mettre en scène la profonde conviction que de le problème posé il n'y pas de solution, mais probablement deux ou même plus » (c'est moi qui traduit).

4. Cf. par ex. les écrits recueillis dans Eco (1990).

5. Je donne entre parenthèses l'indication des pages du roman (Eco 1988). 
6. . Le mouvement du roman est un arc : ce qui se passe est justement ce qui, au début, est refusé, et les personnages tombent dans ce piège qu'ils ont longtemps cherché à éviter. « Da quel giorno - dit Casaubon en parlant de son enfance - incominciai a diventare incredulo. Cioè mi pentii di essere stato credulo. [...] Non è che l'incredulo non debba credere a nulla. Non crede a tutto. Crede a una cosa per volta, e a una seconda solo se in qualche modo discende dalla prima. procede in modo miope, metodico, non azzarda orizzonti. Di due cose che non stiano insieme, crederle tutte e due, e con l'idea che da qualche parte ve ne sia una terza, occulta, che le unisce, questa è la credulità » (47). A savoir, exactement ce qui se passe pour Casaubon et ses copains, au moment où ils sont fervents dans leur reconstruction du Plan des Templiers.

7. . Mais Belbo dit encore: «Spero che non abbia preso la mia teoria per oro colato. Non sto mettendo a posto l'universo. Sto dicendo cosa è un matto per una casa editrice. La teoria è ad hoc, va bene ?». Faut-il le croire?

8. Sur la différence entre lecteur naïf et lecteur critique, cf. Eco (1998).

9. . A la fin de la fabula, mais au début du roman.

10. . Cf. par exemple : «Stavo assaporando le prime gocce di veleno che ci avrebbero portato tutti alla perdizione » (123); "Lentamente smarrii il senso della differenza [...] iniziai a lasciarmi cullare dal sentimento della somiglianza: tutto poteva avere misteriose analogie con tutto » (134-135) ; "Ero diventato uno shaker ambulante" (140); " Mi riusciva sempre più difficile districare il mondo della magia da quello che oggi chiamiamo l'universo della precisione [...] Stavo forse rileggendo la storia intera attraverso gli occhi dei diabolici? » (286) ; « Naturalmente, mi dicevo ritornando a casa, non si tratta di scoprire il segreto dei Templari, ma di costruirlo » (305); «"E allora? Anche noi stiamo costruendo un falso." "E' vero," disse. "Me ne stavo scordando" » (311) ; "Davamo colpi di pollice al Piano che, come creta molle, ubbidiva ai nostri voleri fabulatori » (354); « Era giunto il momento di dare una funzione al Pendolo » (358) ; sino a «mi dissi che la storia era veramente finita » (473).

11. . Ce terme est très fréquent dans le roman: cf. par exemple les pp. 155, 365, 367.

12. .Pendant le rite final au Conservatoire, Agliè dit: «il prigioniero [Belbo] sa troppe cose che nessuno di noi sapeva. Sa persino chi siamo noi, e noi lo abbiamo appreso da lui » (464).

13. . C'est la parodie de Peirce...

14. C'est la parodie de Saussure...

15. Voilà Simmel...

16. Mais l'ordinateur est stupide aussi pour d'autres raisons : parce que « dice solo quello che tutti sanno già » (300); parce que «non crede, non mi fa credere » (190); parce que « il mondo delle macchine cerca di ritrovare il segreto della creazione : lettere e numeri » (205).

17. Prophétique, il serait approprié de dire, au moins si l'on suit l'ordre du récit plutôt que celui de la fabula ; à savoir, si on se pose du côté du lecteur plutôt que de celui des personnages. Si, en fait, la rencontre entre Casaubon et Abulafia a lieu près de la fin de l'histoire, juste avant la nuit au Conservatoire, cet épisode est plutôt placé au début du roman. Les étapes de la progressive reconnaissance ne sont pas les mêmes pour Casaubon et pour le lecteur. C'est justement cette structure temporelle complexe, faite de prolepses et d'analepses, de références croisées, de chevauchements et parfois d'un manque total de chronologie, qui sert à construire une grande partie de la signification du texte du roman. Si la partition de l'espace (Milan, Paris, le Brésil, le Langhe etc.) détermine des divisions «fortes", qui assurent la compréhension textuelle, la séquence de temps - historiques et chronologiques - a un effet, pour ainsi dire, entropique, qui demande beaucoup de coopération au lecteur pour désambiguïser le contenu romanesque. Dans notre cas particulier - celui du lien entre le manque de critères formels dans le raisonnement et la croyance au Plan - les divergences temporelles ont un rôle de premier plan : reconstruire l'ordre de l'histoire signifie en même temps ranger un bon nombre d'étapes logiques et conceptuelles. 


\section{ABSTRACTS}

The aim of this study is to return to the question of the relationship between the plot of the Pendule de Foucault and the hermetic semiosis or the question of irrationalismby nuancing this report, not in the sense of "translation" but of mutual adjustment and partial completion. It is here that "an abstract and figurative theory of stupidity" can constitute a new key to the possible reading of the novel.

Dans cet article, nous proposons une lecture du Pendule de Foucault qui vise à extraire du texte littéraire une théorie de la stupidité et de la raison. Pour le texte de ce roman, il y a plusieurs formes de la bêtise, mais la formulation de cette classification est faite par un personnage ivre et dans un moment très particulier du roman. Ainsi la forme de la narration, celle de la conspiration et la théorie de l'irrationalisme se construisent parallèlement, pendant l'histoire.

INDEX

Keywords: Pendulum, foolishness, stupidity, mutual adjustment, fiction and essay

Mots-clés: Pendule de Foucauld, conspiration, stupidité, traduction, folie

\section{AUTHOR}

\section{GIANFRANCO MARRONE}

Université de Palerme 